\title{
Epistemic and Ontic Quantum Realities
}

\author{
Harald Atmanspacher \\ Institut für Grenzgebiete der Psychologie und Psychohygiene, Freiburg \\ Max-Planck-Institut für extraterrestrische Physik, Garching
}

Hans Primas

ETH-Zentrum, Zürich

Dedicated to Carl Friedrich von Weizsäcker

\begin{abstract}
Quantum theory has provoked intense discussions about its interpretation since its pioneer days. One of the few scientists who have been continuously engaged in this development from both physical and philosophical perspectives is Carl Friedrich von Weizsäcker. ${ }^{1}$ The questions he posed were and are inspiring for many, including the authors of this contribution. Weizsäcker developed Bohr's view of quantum theory as a theory of knowledge. We show that such an epistemic perspective can be consistently complemented by Einstein's ontically oriented position.
\end{abstract}

\section{Introduction}

\subsection{Einstein and Bohr on realism}

Most working scientists believe that there is an external world, which has the status of a reality to be explored by science. The goal of science is to achieve knowledge about how this external world is constituted and develops. Although scientific methodology requires observations and measurements for this purpose, the reality to be described is believed to "exist" independent of its possible empirical accessibility. This view is succinctly formulated by Einstein: ${ }^{2}$

\footnotetext{
"Physics is an attempt conceptually to grasp reality as it is thought independently of its being observed."
}

A realist stance of this kind does not exclude that acts of observation can have effects on an observed entity, and that a measurement of one property of an entity can lead to changes of another property. It simply claims that the "existence" of this entity is in some fundamental sense guaranteed, maybe even necessary as a precondition to observe or measure its properties.

On the other hand, there is an opposing, popular stance to the effect that quantum theory does not admit such an observation-independent realism. This view, which has been perpetuated in many modern monographs and textbooks, goes back to Bohr's claim that in quantum theory a realism with respect to measuring instruments is the only possible realism. According to Bohr: ${ }^{3}$

\footnotetext{
${ }^{1}$ VON WEIZSÄCKER (1985).

${ }^{2}$ Einstein (1949a), p.81.

${ }^{3}$ Quoted in Petersen (1963).
} 
"It is wrong to think that the task of physics is to find out how nature is.

Physics concerns what we can say about nature."

The two quotations by Einstein and Bohr indicate a basic point of disagreement between the two in their ongoing conversations concerning the interpretation of quantum mechanics in the 1920s and 1930s. ${ }^{4}$ Bohr focused on what we could know about and infer from observed quantum phenomena. By contrast, Einstein's position led him to consider Bohr's characterization of quantum theory as incomplete.

Both Einstein and Bohr did not clearly realize that they addressed different concepts of reality. Since they never made their basic viewpoints explicit, it is not surprising that they talked past each other in a number of respects. Both Bohr's operationalistic and Einstein's ontological concept of reality have their proper places in the study of matter. Both are legitimate and even necessary, but they must not be confused with each other. An observation-independent reality must be described in a conceptual and formal framework different from that used for observed facts and measurement.

\subsection{Against classical prejudices}

Quantum features such as non-commutativity, nonlocality, nonseparability and the possibility of entanglement have been forcing us to revise our classical ideas about the nature of matter. For a viable realistic interpretation of quantum theory, the concept of realism must not be associated with ideas taken over from classical physics, such as atomism, localization, separability, or similar preconceptions. Here are three examples.

It is most often assumed that a measurement of a property of a system results in one of the eigenvalues of this property. The underlying idea that every property has a sharp numerical value has been adopted from classical point mechanics without any sensible argument. Hence, an uncritical pointwise valuation of properties should be avoided.

A significant topic in the Bohr-Einstein discussions concerned Einstein's worries about the failure of separability in quantum theory. Yet, according to quantum theory the material world is basically a whole which does not consist of parts. The fact that modern quantum theory can successfully describe many aspects of the behavior of matter in terms of elementary systems and its interactions does not imply that matter is composed of such elementary systems.

The so-called "wave-particle dualism" from the early days of quantum mechanics led to the seemingly ineradicable misunderstanding that an electron is either a particle or a wave. By contrast, the formalism of quantum theory implies that there are infinitely many pairs of states of an electron which are neither wave-like nor particle-like, although they are dual in the same sense as wave-like and particle-like states.

\footnotetext{
${ }^{4}$ Compare Bohr (1949) and Einstein (1949b).
} 


\subsection{Mathematical codification of quantum theory}

The first attempts to formulate quantum mechanics in a mathematically rigorous way were based on the fundamental uniqueness theorem for the irreducible representations of the canonical commutations relations over locally compact phase spaces. ${ }^{5}$ This codification, introduced by von Neumann, ${ }^{6}$ is restricted to quantum systems with only finitely many degrees of freedom and without classical observables. As a consequence, von Neumann's formalism does not comprise a proper description of the interaction of a charged particle with its electromagnetic field, the possibility of symmetry breakings, genuinely irreversible processes, or the existence of molecular (or other) classical observables.

Fortunately, there is no reason to identify quantum theory with the historical Hilbert-space or Fock-space representations. A more comprehensive description has been developed in the language of topological *-algebras. The so-called algebraic quantum theory is nothing other than a straightforward, mathematically precise and complete codification of the heuristic ideas of quantum mechanics of the pioneer days. It uses the same mathematical language for both classical and quantum systems. It is appropriate for microscopic, mesoscopic and macroscopic systems with finitely or infinitely many degrees of freedom. It covers not only classical point mechanics, classical statistical mechanics, and traditional quantum mechanics as special cases, but also systems conceived as fields, such as the electromagnetic field.

Algebraic quantum theory does not make use of any ad hoc assumptions such as hidden variables or quantization procedures as they appear in other approaches. $^{7}$ In the Galilei-relativistic case, the rotation subgroup and the Weyl subgroup, describing space translations and velocity boosts, are all that is needed to generate the canonical commutation relations for position and momentum, for the orbital angular momentum, and for the spin angular momentum. Rather than interpreting quantum theory in terms of concepts of classical physics, the behavior of classical systems has to be explained in terms of quantum theory.

\subsection{Individual and statistical descriptions}

A basic issue for interpretations of quantum theory is the difference between individual and statistical descriptions. For instance, in Bohr's view quantum theory refers to an individual system or an individual experiment, whereas Einstein insisted that quantum theory refers to a statistical ensemble of experiments rather than individual systems. Is it possible to resolve this issue within algebraic quantum theory?

In classical mechanics, the mathematical formalism required for an individual description is different from the formalism required for a statistical descrip-

\footnotetext{
${ }^{5}$ Stone (1930), von Neumann (1931).

${ }^{6}$ VON NEUMANN (1932).

${ }^{7}$ We do not consider Bohmian mechanics, the consistent history proposal, the many-worlds view, the approach by Ghirardi, Rimini, Weber and Pearle, and related approaches, since we regard them as nonviable.
} 
tion. While the individual description of point mechanics is usually formulated in terms of a (generally nonlinear) dynamics on an even-dimensional smooth manifold as a phase space, the statistical formulation uses Koopman's Hilbertspace formalism with a linear dynamics. ${ }^{8}$

This example strongly suggests that the traditional Hilbert-space codification of quantum mechanics corresponds to Koopman's Hilbert-space formalism of classical mechanics. Their common statistical nature is reflected by the fact that they are both formulated in terms of the $\mathrm{W}^{*}$-algebraic probability theory. On the other hand, the algebraic description of individual physical systems does not use the topological structure of a $\mathrm{W}^{*}$-algebra, ${ }^{9}$ but only the structure of $\mathrm{C}^{*}$-algebras with its algebraically defined norm topology.

\section{Distinguishing Epistemic and Ontic Perspectives}

\subsection{Epistemology and ontology}

A crucial issue of any interpretational approach with respect to a scientific theory is the relation between elements of the theory on the one hand and elements of the domain of reality for which the theory is designed on the other. This so-called relation of reference cannot be exhaustively addressed within the scope of the scientific discipline concerned. For its proper discussion, genuinely philosophical issues must be taken into account explicitly.

One of the most general demands on a sound philosophical discussion is the distinction between epistemological and ontological statements. While ontology is a branch of metaphysics, epistemology comprises all kinds of issues related to the knowledge (or ignorance) of information gathering and using systems. The ways in which human beings process information (perceptually, cognitively, and otherwise), thus represent a set of central epistemological questions.

Ontology refers to the nature and behavior of systems as they are, independent of any empirical access. The different stances of Einstein and Bohr can thus be related to an ontological versus an epistemological emphasis. Their conflicting viewpoints demonstrate that particular problems in physics (as well as other natural sciences) make it important to distinguish the two perspectives.

For a proper discussion of interpretations of quantum theory, Scheibe introduced the notions of epistemic and ontic states of a system rather than addressing epistemological and ontological issues in the more general sense mentioned above. ${ }^{10}$ Discussing epistemic and ontic states of a system can be an epistemological or an ontological matter. For instance, referring to epistemic and ontic states of a system just as they are might be understood ontologically. Referring

\footnotetext{
${ }^{8}$ KoOpman (1931).

${ }^{9} \mathrm{~A} \mathrm{~W}^{*}$-algebra $\mathfrak{M}$ is a $\mathrm{C}^{*}$-algebra which is the dual Banach space of a Banach space $\mathfrak{M}_{*}$. The topology relevant for the $\mathrm{W}^{*}$-algebraic probability theory is the $\sigma\left(\mathfrak{M}, \mathfrak{M}_{*}\right)$-topology on $\mathfrak{M}$.

${ }^{10}$ SCheibe (1964), Scheibe (1973).
} 
to descriptions in terms of epistemic and ontic states, briefly epistemic and ontic descriptions, might be understood epistemologically.

\section{$2.2 \quad$ Epistemic and ontic descriptions}

States of a system to which epistemic descriptions refer are called epistemic states. The mathematical representation of such states encodes empirically obtainable knowledge about them. If the knowledge about states and their associated properties is expressed by probabilities in the sense of relative frequencies for a statistical ensemble of independently repeated experiments, we speak of a statistical description and of statistical states.

Insofar as epistemic descriptions refer to a state concept encoding knowledge, they depend on observation and measurement. Such kinds of empirical access necessarily introduce a context under which a system is investigated. Therefore, properties associated with an epistemic state are contextual. General fundamental principles cannot be expected within epistemic descriptions.

States of a system to which ontic descriptions refer are called ontic states. They represent the system exhaustively, i.e. an ontic state is "just the way it is", without any reference to epistemic knowledge or ignorance. In this sense, ontic states are empirically inaccessible. The properties of the system are understood as intrinsic properties. As individual states, ontic states are the referents of individual descriptions.

The goal of an ontic description of quantum systems is not a nostalgic desire for a classical picture of reality behind quantum phenomena, but to understand quantum theory as a fundamental theory of the material world, based on first principles such as symmetries. Such principles are assumed to be quite universally valid, and they are used to provide a description of the material world which is as context-independent as possible or reasonable. We do not propose an ontic description as an absolutely context-free, ultimately fundamental theory of matter.

To the same degree to which ontic descriptions are context-independent, they hide the richness and variety of empirical reality. This manifests itself in the fact that pattern detection and recognition devices determine what is considered as relevant or irrelevant. Patterns are detected and recognized by rejecting information which is selected as irrelevant in particular contexts. Based on such contexts, an epistemic state refers to the knowledge that can be obtained about an ontic state.

\subsection{Interplay of epistemic and ontic approaches}

The very characterization of epistemic descriptions in terms of empirical access implies that tools of observation and measurement must be addressed. This requires the description of engineering instruments and the possibility of unambiguous communication. If these tools are taken for granted, their states have to be conceived as ontic states. As a consequence, any epistemic description presupposing tools of empirical access must be understood relative to an ontic 
description of these tools. In this sense, any epistemic description requires an ontological commitment.

Accordingly, both epistemic and ontic elements are involved already at a fairly basic level of discussion. In order to avoid confusion, corresponding concepts of epistemic and ontic states need to be distinguished. If this is not taken care of, there is a risk of confounding issues belonging to categorially different domains of discussion. As Fetzer and Almeder emphasize, ${ }^{11}$

$$
\begin{gathered}
\text { "an ontic answer to an epistemic question (or vice versa) } \\
\text { normally commits a category mistake." }
\end{gathered}
$$

For instance, it would be a category mistake to discuss an ontic description of an observation-independent reality in terms of measurements, and it would be a category mistake to discuss the epistemic description of the observation of facts in terms of theories not involving measurement as a key topic.

Drawing the distinction between epistemic and ontic descriptions does not imply, though, that the two categories are unrelated to each other. On the contrary, the crucial point is about the relationship between the two frameworks rather than the selection of one at the expense of the other. Based on an ontic description of a physical system, epistemic descriptions can be derived by additionally specifying contexts which distinguish relevant from irrelevant patterns of empirical reality. This distinction can be mathematically accomplished by introducing a contextual topology. One ontic description can give rise to numerous physically inequivalent epistemic descriptions.

\section{Epistemic Descriptions of Quantum Systems}

\subsection{Every experiment requires a Boolean context}

In physics, epistemic descriptions deal with experiments and measurements. It is a basic fact of experimental physics that the registration of experimental results is described in terms of classical engineering science. This situation requires a Boolean domain of discourse, corresponding to Bohr's insight: ${ }^{12}$

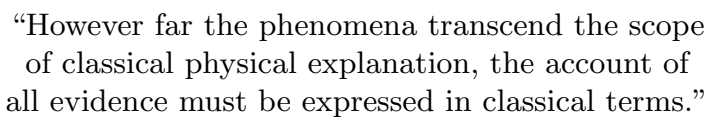

From a modern point of view, "classical" is to be interpreted as "Boolean", not as macroscopic. Whether a system shows quantal or classical behavior is independent of its size. There are small molecular systems with classical properties, like the chirality of most biologically important molecules. Furthermore, measuring tools are not necessarily macroscopic. For example, molecular genetic coding realizes a highly reliable classical, irreversible and nonanticipating measuring instrument of molecular size.

\footnotetext{
${ }^{11}$ Fetzer \& Almeder (1993), p.101.

${ }^{12}$ Bohr (1949), p.209.
} 


\subsection{Von Neumann's statistical formulation}

The first mathematically rigorous codification of the ideas of the pioneers of quantum mechanics was given by von Neumann. ${ }^{13}$ He derived the Hilbert-space representation for the states of quantum systems from the uniqueness theorem by Stone and von Neumann. ${ }^{14}$ This theorem says that for finitely many degrees of freedom there is (up to unitary equivalence) only one irreducible Hilbertspace representation of the fundamental canonical commutation relations. The restriction of von Neumann's codification to systems with only finitely many degrees of freedom is a severe limitation which excludes an explicit description of the environment (like the radiation field) or of measuring tools.

The Hilbert-space quantum theory introduced by von Neumann is a statistical theory which expresses the expectation value $\mathcal{E}_{t}(A)$ at time $t$ of an observable $A$ on a Kolmogorov probability space $\left(\boldsymbol{\Lambda}, \boldsymbol{\Sigma}, \boldsymbol{\mu}_{t}\right)$ as $\mathcal{E}_{t}(A)=\int_{\boldsymbol{\Lambda}} \lambda \boldsymbol{\mu}_{t}(d \lambda)$. Here $A$ is a selfadjoint Hilbert-space operator with the spectral resolution $A=$ $\int_{\Lambda} \lambda E(d \lambda)$, where $\mathcal{B} \mapsto E(\mathcal{B})$ is a projection-valued set function on the Boolean $\sigma$-algebra $\boldsymbol{\Sigma}$ of Borel sets $\mathcal{B}$ in the spectrum $\boldsymbol{\Lambda}$ of $A$. The probability measure $\boldsymbol{\mu}_{t}$ is given by $\boldsymbol{\mu}_{t}(\mathcal{B})=\operatorname{tr}\left\{D_{t} E(\mathcal{B})\right\}$, where the density operator $D_{t}$ (a nonnegative trace-class operator with $\operatorname{tr}\left(D_{t}\right)=1$ ) describes the statistical state at the instant $t$.

A statistical description refers to an ensemble of experimental results as registered with laboratory instruments. It may be tempting to interpret such an ensemble as a collection of individual systems. However, in quantum theory object systems are in general entangled with their environment. Therefore, a statistical description does not allow us to refer to properties of individual quantum objects. This does not mean that quantum systems do not "have" properties, but it means that the discussion of properties of individual quantum systems does not pertain to the domain of epistemic descriptions.

In von Neumann's approach, statistical states are represented as linear functionals, generalizing the idea that the expectation value of a sum of operators should equal the sum of the expectation values of individual operators (even if they do not commute). ${ }^{15}$ This postulate is difficult to justify since the sum of non-commuting operators is not operationally explained. It is not at all trivial that statistical expectation values can be represented by linear functionals. This problem has been solved by Gleason's theorem, ${ }^{16}$ which implies that every $\sigma$-additive probability measure on the projection lattice on a Hilbert space $\mathcal{H}$ of dimension larger than two can be extended to a unique normalized positive normal linear functional $\mathfrak{B}(\mathcal{H}) \rightarrow \mathbb{C}$ on the algebra $\mathfrak{B}(\mathcal{H})$ of all bounded linear operators acting on $\mathcal{H}$. In quantum theory, such a normalized positive normal linear functional is called a statistical state functional. Gleason's theorem is of probabilistic nature and does not apply to individual descriptions.

In addition to the linearity of statistical state functionals, the evolution of

\footnotetext{
${ }^{13}$ Von Neumann (1932).

${ }^{14}$ Stone (1930), von Neumann (1931).

${ }^{15}$ Postulate E in von Neumann (1932), chapter IV.1, p.164.

${ }^{16}$ Gleason (1957).
} 
statistical states is linear as well. In contrast to widespread misconceptions, this linearity is independent of the superposition principle. Physical statistical theories are based on abstract convex structures which can be defined without any reference to linear spaces. ${ }^{17} \mathrm{~A}$ basic postulate for any statistical description is Mackey's axiom IX requiring the commutativity of the operation of convex linear combinations with the the time evolution semigroup for a statistical ensemble. ${ }^{18}$ It is essentially this postulate which implies the linearity of the dynamics of any fundamental statistical theory. ${ }^{19}$

A well-known example is Koopman's Hilbert-space formalism which rephrases the nonlinear Hamiltonian equations of motion of classical mechanics in terms of linear equations of motion for classical statistical mechanics. ${ }^{20}$ Another example is the equivalence of nonlinear stochastic differential equations in the sense of Itô with the linear Fokker-Planck equations.

\subsection{The so-called measurement problem}

To include measurements in his statistical approach, von Neumann introduced the ad hoc postulate that a measurement of an observable $A$ with a purely discrete nondegenerate spectrum and the spectral resolution $A=\sum_{k} a_{k} P_{k}$ $\left(P_{k}=P_{k}^{2}=P_{k}^{*}\right)$ transforms any initial density operator $D$ into the eigenstate $D_{j}:=P_{j} D P_{j} / \operatorname{tr}\left(D P_{j}\right)$ if the eigenvalue $a_{j}$ is measured. ${ }^{21}$ The nonlinear, nondeterministic, discontinuous, instantaneous change $D \rightarrow D_{j}$ formalizes the historical idea of "quantum jumps" and is usually referred to as the projection postulate (corresponding to the reduction of the wave packet). Since the density operator $D$ always refers to a statistical ensemble rather than one particular outcome, von Neumann's discontinuous nonlinear map $D \rightarrow D_{j}$ describes a change from a statistical to an individual description.

In classical probability theory such a map can be expressed in terms of Bayes' rule for updating probabilities. That is, $\operatorname{tr}\left(D_{j} A\right)$ is the conditional expectation of $A$, under the condition that the event $a_{j}$ has occurred. Since the projection postulate merely indicates a change from a statistical to an individual description, the interpretation of the nonlinear map $D \rightarrow D_{j}$ as a physical process is untenable. In the statistical description, the so-called objectification problem ("how can a pointer observable assume a definite value?") is ill-posed as well: a statistical description never leads to definite individual results, but to well-defined probabilistic predictions.

\subsection{Contemporary views on measurement}

A realistic theory of measurements has to be dynamical and, therefore, cannot be based on von Neumann's projection postulate. Moreover, the dated view that

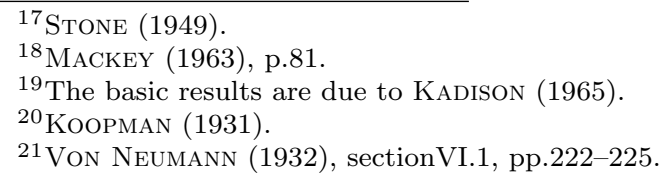


quantum measurements deal with the determination of eigenvalues of selfadjoint operators (the so-called "observables") has been superseded by modern response theory. It specifies a classical output signal of a quantum system in response to a classical external stimulus, say an electromagnetic field or a mechanical force. For example, no spectroscopic measurement is a direct measurement of energy. The response in a spectroscopic experiment is given in terms of a molecular electric or magnetic multipole moment. Only by using the intricate theory of line broadening and level shifts can approximate information about energy levels be deduced.

A mathematical model which relates the information about an input stimulus to the information contained in the output response is called an information channel. Thus, information is a central concept of any epistemic description. The output system can be characterized by a Kolmogorov probability space $(\boldsymbol{\Omega}, \boldsymbol{\Sigma}, \boldsymbol{\nu})$, consisting of a set $\boldsymbol{\Omega}$ (the sample space), a class $\boldsymbol{\Sigma}$ of subsets of $\boldsymbol{\Omega}$ which is a $\sigma$-algebra (of experimentally decidable events), and a $\sigma$-additive probability measure $\boldsymbol{\nu}$ on $\boldsymbol{\Sigma}$. The classical statistical output states are given by probability densities, i.e. by positive normalized elements of the Banach space $\boldsymbol{L}^{1}(\boldsymbol{\Omega}, \boldsymbol{\Sigma}, \boldsymbol{\nu})$ of equivalence classes of integrable complex-valued functions on $\boldsymbol{\Omega}$.

Within the traditional Hilbert-space framework, a quantum information channel can be described in terms of a Hilbert space $\mathcal{H}$ and an affine map $\Lambda: \mathfrak{B}(\mathcal{H})_{*} \rightarrow L^{1}(\boldsymbol{\Omega}, \boldsymbol{\Sigma}, \boldsymbol{\nu})$ which transfers a normal state functional $\rho \in \mathfrak{B}(\mathcal{H})_{*}$ on the $\mathrm{W}^{*}$-algebra $\mathfrak{B}(\mathcal{H})$ of all bounded operators on $\mathcal{H}$ to a probability density $f \in L^{1}(\boldsymbol{\Omega}, \boldsymbol{\Sigma}, \boldsymbol{\nu})$ of the classical output system, $\Lambda(\rho)=f .^{22}$ The statistical state functional $\rho$ can also be represented by a density operator $D_{\rho}$ acting on $\mathcal{H}, \rho(A)=\operatorname{tr}\left(D_{\rho} A\right)$ for every $A \in \mathfrak{B}(\mathcal{H})$. Every affine mapping from the set of all density operators on the Hilbert space $\mathcal{H}$ to the set of probability measures on the measurable space $(\boldsymbol{\Omega}, \boldsymbol{\Sigma})$ can be realized by a normalized positive operator-valued measure $F: \boldsymbol{\Sigma} \rightarrow \mathfrak{B}(\mathcal{H})$, also called a probability-operator measure. ${ }^{23}$ Every positive operator-valued measure $F: \boldsymbol{\Sigma} \rightarrow \mathfrak{B}(\mathcal{H})$ defines by $\boldsymbol{\mu}(\mathcal{B})=\operatorname{tr}\left\{D_{\rho} F(\mathcal{B})\right\}(\mathcal{B} \in \boldsymbol{\Sigma})$ a probability measure $\boldsymbol{\mu}$ on the measurable space $(\boldsymbol{\Omega}, \boldsymbol{\Sigma})$, and by $\boldsymbol{\mu}(\mathcal{B}):=\rho\{F(\mathcal{B})\}$ and $\Lambda(\rho):=f:=d \boldsymbol{\mu} / d \boldsymbol{\nu}$ a measurement channel $\Lambda(\rho)$.

Applying standard methods of statistical decision theory, information about the statistical state $\rho$ of the object system can be inferred by observing the classical output system. Since the convex set of all statistical quantum states is in general not a simplex, a statistical description of quantum systems does not determine an ensemble of individual systems. That is, a description of a quantum system in terms of statistical, epistemic states does not allow a reconstruction of an individual description in terms of individual, ontic states. A statistical experiment yields information about the epistemic quantum state which determines the response behavior of the chosen experimental arrangement. Any statement about intrinsic "properties" of an individual object system is impossible in an epistemic description.

\footnotetext{
${ }^{22}$ This fertile view was first proposed by HoLEvo (1972).

${ }^{23}$ For details of the definition of positive operator-valued measures compare for example BERBERIAN (1966).
} 


\subsection{Full-fledged epistemic descriptions}

Essentially all verifications and applications of quantum theory are based on the engineering approach sketched in the preceding subsection. Nevertheless, this approach is not complete. Engineering quantum theory cannot not explain the existence of facts, and it does not specify under which experimental conditions measurable events can occur at all. It provides just the conditional probability for an event $\mathcal{B} \in \boldsymbol{\Sigma}$ registered on a classical output device, under the condition that the measuring instrument has factually and irreversibly registered this event. To achieve a complete dynamical description of the measurement process, the tools necessary for a registration of the results of a measurement have to be included in the theoretical description.

Facts make particular propositions true and others false, so they are to be described in Boolean terms. This means that in quantum theory facts must be described by classical observables. They are defined via the center of the algebra of the full quantum theoretical description. The center $\mathfrak{Z}(\mathfrak{M})$ of an algebra $\mathfrak{M}$ is a commutative algebra, defined as the set of all elements of $\mathfrak{M}$ which commute with all elements of $\mathfrak{M}$. The selfadjoint elements of the center $\mathfrak{Z}(\mathfrak{M})$ of the algebra of observables of a quantum theoretical description are called classical observables. $^{24}$

The emergence of classical observables can only be derived from quantum systems with infinitely many degrees of freedom, e.g. including the electromagnetic field. This is impossible under the condition of a locally compact phase space presupposed by the Stone-von Neumann theorem of traditional Hilbertspace quantum mechanics. If this condition is relaxed, the framework of algebraic quantum theory allows us to comprise classical observables.

A full statistical description for the measurement of an observable $A$ with a purely discrete nondegenerate spectrum and the spectral resolution $A=$ $\sum_{k} a_{k} P_{k}$ is given by the linear map $D \rightarrow \sum_{k} p_{k} D_{k}$, with $p_{k}:=\operatorname{tr}\left(D P_{k}\right)$. This map $D \rightarrow \sum_{k} p_{k} D_{k}$ is a statistical description of a measuring operation if and only if the final density operator $\sum_{k} p_{k} D_{k}$ represents a mixture of classically disjoint states with the weights $p_{k}$. Two quantum states described by two density operators $D_{r}$ and $D_{s}$ are called disjoint, if a classical observable $Z$ exists such that $\operatorname{tr}\left(Z D_{r}\right) \neq \operatorname{tr}\left(Z D_{s}\right)$. Hence, disjoint states can be distinguished and classified operationally by the numerical value of an appropriate classical observable. While a nonpure reduced state of a quantum system entangled with its environment can be described by $p_{1} D_{1}+p_{2} D_{2}+\cdots p_{n} D_{n}$, a proper classical mixture is represented as a density operator by the expression $p_{1} \mathcal{D}_{1} \oplus p_{2} \mathcal{D}_{2} \oplus \cdots \oplus p_{n} \mathcal{D}_{n}$, where $D_{1}:=\mathcal{D}_{1} \oplus 0 \oplus \cdots \oplus 0$, etc.

A quantum system can act as a measurement apparatus only if it can produce disjoint final states. If the fundamental dynamics is given by a one-parameter group of automorphisms, such a result cannot be completed in finite time. But Hepp proved that for appropriate quantum systems a one-parameter automorphism group exists such that for classically equivalent initial states their asymp-

\footnotetext{
${ }^{24}$ By construction, classical observables depend on Planck's constant $\hbar$. The still widely held view that classical mechanics is given by the limit $\hbar \rightarrow 0$ is untenable.
} 
totic limits exist for $t \rightarrow \infty$ and their final states are disjoint. ${ }^{25}$

Hepp's crucial contribution did not solve the statistical measurement problem, though, since it leaves the possibility to reverse the measurement process at any finite time. ${ }^{26}$ Since facts cannot be undone, a measuring process has to be governed by an irreversible dynamics. The decisive idea of combining the nonanticipative character of laboratory instruments with a dynamics generating asymptotically disjoint final states was worked out by Lockhart and Misra. ${ }^{27}$ In the proposed dynamics the time-inversion symmetry is broken and given by a weakly contractive semigroup (based on a K-flow) which produces asymptotically disjoint final states in a strictly irreversible manner. The emergence of disjoint states occurs progressively over finite amounts of time. ${ }^{28}$

\section{Ontic Descriptions of Quantum Systems}

\subsection{General structure of individual descriptions}

Quantum theory is well established only in its statistical epistemic formulations. Although Bohr defended the view that quantum theory refers to individual systems, he never indicated a mathematical formulation of his conviction. Most adherents of one or another version of the "Copenhagen interpretation" use von Neumann's statistical codification, which does not allow a description of individual systems or experiments.

Statistical descriptions of classical systems can be given in terms of Boolean algebras. In Hilbert-space quantum theory, the corresponding probabilistic structure is given by the non-Boolean lattice of closed subspaces of a Hilbert space. A key feature of this lattice is that it is partially Boolean. That is, it can be represented as a family of Boolean algebras pasted together such that their operations agree with each other wherever two or more Boolean algebras overlap. $^{29}$ In the following, we adopt a similar structure for individual nonprobabilistic descriptions. Here, classical systems are characterized by commutative algebras, while quantum systems are described by non-commutative, but partially commutative algebras.

\subsection{Algebraic framework for individual descriptions}

The paradigmatic example for a non-probabilistic description of individual systems is classical point mechanics, where a state of an individual system at time $t$ can be represented by a point $\boldsymbol{\gamma}_{t} \in \boldsymbol{\Gamma}$ of a locally compact phase space $\boldsymbol{\Gamma}$. A

${ }^{28}$ It has been objected that a measurement process with asymptotically disjoint final states implies an infinite measurement time. This is a misunderstanding: every measurement in engineering physics is of this type. For details, compare Primas (1997), Primas (2000).

${ }^{29}$ For details, compare Kochen \& Specker (1965), Kochen \& SpeCKer (1967).
} 
fully equivalent algebraic description can be given in terms of commutative $\mathrm{C}^{*}$ algebras. The Gelfand representation of commutative Banach algebras implies that the commutative algebra $\mathcal{C}_{0}(\boldsymbol{\Gamma})$ of all continuous complex-valued functions on $\boldsymbol{\Gamma}$ vanishing at infinity is a commutative $\mathrm{C}^{*}$-algebra $\mathfrak{C}$, and every commutative $\mathrm{C}^{*}$-algebra can be represented as a function algebra $\mathcal{C}_{0}(\boldsymbol{\Gamma})$ where $\boldsymbol{\Gamma}$ is some locally compact space. In the algebraic description of classical systems with the underlying commutative $\mathrm{C}^{*}$-algebra $\mathfrak{C}$, every individual state is represented by a multiplicative linear functional $v_{t}$ on $\mathfrak{C}$.

For individual quantum systems, the role of the phase space in classical mechanics is taken over by a unital simple non-commutative $\mathrm{C}^{*}$-algebra $\mathfrak{A}$. The partially commutative structure of a quantum system is reflected by the requirement that every commutative $\mathrm{C}^{*}$-subalgebra $\mathfrak{C} \subset \mathfrak{A}$ represents an individual classical system. The singly generated (by the unit element $\mathbf{1}$ and a single selfadjoint element $A \in \mathfrak{A}$ ) commutative $\mathrm{C}^{*}$-subalgebras of $\mathfrak{A}$ represent the intrinsic properties of the system.

The ontic state of an individual system refers to its mode of being at a given instant. The required partially commutative structure implies that an ontic state has to be represented by a functional $v: \mathfrak{A} \rightarrow \mathbb{C}$ which is a linear positive functional on every commutative $\mathrm{C}^{*}$-subalgebra of $\mathfrak{A}$. Yet, for individual descriptions there is no reason to assume that states are represented by positive linear functionals on the full $\mathrm{C}^{*}$-algebra $\mathfrak{A}$. (Recall that in epistemic descriptions the linearity of state functionals is warranted by the probabilistic Gleason theorem.)

As a natural condition for ontic state functionals, Misra proposed monotone positivity: ${ }^{30}$ A functional $v$ on a $\mathrm{C}^{*}$-algebra $\mathfrak{A}$ is called monotone positive if and only if $A \geq B$ implies $v(A) \geq v(B) \geq 0$ for all positive elements $A, B$ in $\mathfrak{A}$. While every positive linear functional is monotone positive, the converse is not true. It is an open question whether nonlinear state functionals are necessary for individual descriptions of quantum systems, or what special role linear state functionals play.

\subsection{Ontic valuations of intrinsic properties}

In classical point mechanics an ontic state is determined by the values which its associated intrinsic properties have at a given instant. This attribution of a dispersion-free numerical value to every physical property is an ontic valuation of the property by a real-valued point function $v: \mathfrak{A} \rightarrow \mathbb{R}$. This valuation is continuous in the sense that for a given ontic state small variations of any property lead to small variations of its valuation.

In the early days of quantum mechanics, Dirac proposed that a dynamical variable $A$ has the value $a$ if the state vector $\Psi$ is the eigenvector of $A$ with the eigenvalue $a .^{31}$ This partial ontic valuation of properties by point functions, which is taken over from classical point mechanics, is inappropriate for

\footnotetext{
${ }^{30}$ Misra (1967).

${ }^{31}$ DiRAC (1926), p.666.
} 
quantum mechanics. The principal objection against Dirac's valuation is that it is not continuous: Noncommuting elements cannot be valuated in a continuous manner by point functions. For example, in traditional Hilbert-space quantum mechanics, for every projection $F=F^{*}=F^{2}$ and $\varepsilon>0$ there exists another projection $F_{\varepsilon}$ with $\left\|F-F_{\varepsilon}\right\|=\varepsilon$ which does not commute with $F$. If $\Psi$ is an eigenvector of $F$ with the eigenvalue 1 , then $F$ has the value 1 according to Dirac's proposal, while the projection $F_{\varepsilon}$ cannot be valued.

In 1935, Schrödinger proposed, in a qualitative way, a continuous ontic valuation of intrinsic properties in terms of a "blurring of all variables" and emphasized that this is a perfectly clear and consistent concept. ${ }^{32}$ Since the valuation proposed by Schrödinger has nothing to do with missing information, uncertainty, inexactness, imprecision, indefiniteness, ambiguity, randomness, or probability, we refer to it simply as a valuation by set functions.

An intrinsic property of an individual quantum system is represented by a commutative $\mathrm{C}^{*}$-subalgebra $\mathfrak{C} \subset \mathfrak{A}$ generated by a single selfadjoint element $C \in \mathfrak{A}$ with the spectrum $\operatorname{sp}\{C\} \subset \mathbb{R}$. Then $\mathfrak{C}$ is isometric *-isomorphic to the function algebra $\mathcal{C}(\operatorname{sp}\{C\})$. An ontic valuation of the intrinsic property $C$ can be characterized by the continuous set function $\mathcal{B} \mapsto \boldsymbol{\mu}_{t}^{\mathfrak{C}}(\mathcal{B})$, where $\mathcal{B}$ is an element of the $\sigma$-algebra of Borel sets of the spectrum $\operatorname{sp}\{C\}$ of $\mathfrak{C} \sim \mathcal{C}_{0}(\operatorname{sp}\{C\})$. Here the measure $\boldsymbol{\mu}_{t}^{\mathfrak{C}}$ is defined via the Bochner-Cramér representation of the characteristic function

$$
s \mapsto v_{t}\left\{e^{i s C}\right\}=\int_{\operatorname{sp}\{C\}} e^{i s x} \boldsymbol{\mu}_{t}^{\mathfrak{C}}(d x) \quad, \quad s \in \mathbb{R},
$$

where $v_{t}$ is the ontic state at time $t$. In both classical and quantum theories, every intrinsic property of an isolated or open system has a uniquely defined continuous ontic valuation with respect to every ontic state. Moreover, the ontic valuation of properties is in no way probabilistic. It refers to properties, which the system actually and objectively has, and not to potentialities, propensities, or dispositions. Therefore, ontic valuations should not be misinterpreted as fluctuations or statistical spreads of supposed valuations by point functions.

If an individual quantum system is entangled with its environment, then its ontic state cannot be extremal, and no intrinsic property has a pointwise valuation, $v\left(C^{2}\right)>v(C)^{2}$, or equivalently, $\int_{\operatorname{sp}\{C\}} x^{2} \boldsymbol{\mu}_{t}^{\mathfrak{C}}(d x)>\left(\int_{\operatorname{sp}\{C\}} x \boldsymbol{\mu}_{t}^{\mathfrak{C}}(d x)\right)^{2}$.

\subsection{Ontically dependent properties}

The non-commutativity of the $\mathrm{C}^{*}$-algebra underlying an ontic, individual description of a quantum system exhibits a new kind of dependence of properties. Loosely speaking, two properties represented by the commutative $\mathrm{C}^{*}$-algebras $\mathfrak{C}_{1}$ and $\mathfrak{C}_{2}$ are called ontically independent if every possible ontic valuation of

\footnotetext{
${ }^{32}$ SCHRÖDINGER (1935), pp.811-812. This idea of an "objective vagueness" has not become popular but is endorsed by some contemporary philosophers and physicists under terms like "Verschmiertheit", "properties lacking sharp values", "blurred variables", "ontic blurring", "objective fuzziness", "unsharp properties".
} 
$\mathfrak{C}_{1}$ is compatible with every possible ontic valuation of $\mathfrak{C}_{2}$. A trivial requirement for the ontic independence of two properties is that they are functionally independent, i.e. that they cannot be commonly represented by a third one.

Accordingly, two properties represented by the commutative $\mathrm{C}^{*}$-algebras $\mathfrak{C}_{1}$ and $\mathfrak{C}_{2}$ are called ontically dependent in a nontrivial way, if they are functionally independent, $\mathfrak{C}_{1} \cap \mathfrak{C}_{2}=\mathbf{1} \mathbb{C}$, and if at least two ontic valuations $v_{1}$ and $v_{2}$ exist such that there is no ontic valuation $v$ extending both $v_{1}$ and $v_{2}$. Two intrinsic properties are called incompatible if they are functionally independent but ontically dependent.

Consider two selfadjoint elements $C_{1}$ and $C_{2}$ of a simple non-commutative $\mathrm{C}^{*}$-algebra $\mathfrak{A}$ with $C_{1} C_{2} \neq C_{2} C_{1}$. There exist two pure linear functionals $v_{1} \in$ $\mathfrak{A}^{*}$ and $v_{2} \in \mathfrak{A}^{*}$ such that $v_{1}\left(C_{1}^{2}\right)=v_{1}\left(C_{1}\right)^{2}$ and $v_{2}\left(C_{2}^{2}\right)=v_{2}\left(C_{2}\right)^{2}$. As proven by Misra, a simple $\mathrm{C}^{*}$-algebra $\mathfrak{A}$ admits a valuation by a monotone positive functional $v$ which is dispersion-free, $v\left(A^{2}\right)=v(A)^{2}$, for all selfadjoint elements $A \in \mathfrak{A}$, if and only if $\mathfrak{A}$ is commutative. ${ }^{33}$ Consequently, there is no valuation $v$ with the property $v\left(C_{1}^{2}\right)=v\left(C_{1}\right)^{2}$ and $v\left(C_{2}^{2}\right)=v\left(C_{2}\right)^{2}$ if $C_{1} C_{2} \neq C_{2} C_{1}$. That is, mutually non-commuting commutative $C^{*}$-subalgebras of a $C^{*}$-algebra represent incompatible properties.

\subsection{Superposition principle and ontic entanglement}

There are many physically equivalent representations of quantum theory. The traditional irreducible Hilbert-space representation has the important advantage that it allows a very simple mathematical formulation of the quantum mechanical superposition principle in term of state vectors: Every linear combination of two state vectors is an other state vector, called a coherent superposition of the two generating state vectors. Since pure states are represented by rays (and not by vectors), the underlying state space is the set of rays, i.e. a nonlinear projective space. As a consequence, the linearity of the traditional formulation of the quantum mechanical superposition principle is not an intrinsic feature, but a peculiarity of a cleverly chosen representation. From a conceptual point of view, a representation-independent formulation without linear structure is preferable: Any two different pure states generate an uncountably infinite family of mutually different pure states different from the two generating pure states. ${ }^{34}$

The meaning of a superposition state is not that a system "can be in two or more states at the same time", but that there is an ontic holistic entanglement, implying that the system considered is not composed of independently existing parts. Consider for example two subsystems described by mutually commuting $\mathrm{C}^{*}$-subalgebras $\mathfrak{B} \subset \mathfrak{A}$ and $\mathfrak{C} \subset \mathfrak{A}$ of an individual quantum system described by the $\mathrm{C}^{*}$-algebra $\mathfrak{A}$. Consider any linear state functional $v$ on $\mathfrak{A}$, two arbitrary elements $B_{j} \in \mathfrak{B}$ with $\mathbf{0} \leq B_{j} \leq \mathbf{1}$, and two arbitrary elements $C_{k} \in \mathfrak{C}$ with $\mathbf{0} \leq C_{k} \leq \mathbf{1}, B_{j} C_{k}=C_{k} B_{j}$, with $j, k=1,2$. A quantity describing the mutual ontic dependence of the two commuting subsystems is given by the functional

\footnotetext{
${ }^{33}$ MisRa (1967).

${ }^{34}$ For a representation-independent mathematical formulation of quantum mechanical superpositions, see RoBerts \& ROEPSTORFF (1969).
} 
$\kappa:=\frac{1}{2} v\left(B_{1} C_{1}+B_{1} C_{2}+B_{2} C_{2}-B_{2} C_{1}\right)$. Then Schwarz's inequality implies ${ }^{35}$

$$
|\kappa|^{2} \leq 1+\frac{1}{4} v\left\{\left(B_{1} B_{2}-B_{2} B_{1}\right)\left(C_{1} C_{2}-C_{1} C_{2}\right)\right\} \leq 2 .
$$

If $\mathfrak{B}$ or $\mathfrak{C}$ is commutative, we get $|\kappa| \leq 1$, a generalized Bell inequality. It is well known that in quantum theory Bell's inequality can be violated. If the generalized Bell inequality is violated, $|\kappa|>1$, the state functional $v$ is holistically entangled with respect to the decomposition $(\mathfrak{B}, \mathfrak{C})$. The relation $|\kappa|^{2} \leq 1+\frac{1}{4} v\left\{\left(B_{1} B_{2}-B_{2} B_{1}\right)\left(C_{1} C_{2}-C_{1} C_{2}\right)\right\}$ shows that ontic holistic entanglement between two subsystems is possible if and only if there exist incompatible properties in both subsystems.

\subsection{Dynamics of individual quantum systems}

The usual time-dependent Schrödinger equation is linear since it refers to a statistical description. This fact, however, does not imply the linearity of the state dynamics in individual descriptions. Since the quantum mechanical superposition principle is independent of any dynamical principle, a nonlinear dynamics for individual quantum states is not in contradiction with the quantum mechanical superposition principle. In particular, the quantum mechanical superposition principle does not require the invariance of superpositions under the time evolution.

According to current knowledge, the only way to derive a dynamics from fundamental first principles is to introduce, first, group-theoretical elementary systems and, then, interactions by gauge fields. Such a procedure is not satisfactory because elementary systems also represent the sources of the fields by which they interact.

Nevertheless, bare elementary systems are defined as ergodic representations of the presupposed kinematical group. Both the Lorentz and the Galilei group are linear Lie groups, implying that the dynamics of the corresponding bare elementary systems is governed by linear equations of motion. Yet, bare elementary systems are just auxiliary constructions. They are transformed to dressed systems by interactions with gauge fields (e.g. electromagnetism, gravitation). Dressing is a rather complicated procedure which may lead to nonlinear equations of motion, in particular due to self-interactions. Moreover, the inclusion of gauge fields enforces a discussion of quantum systems with infinitely many degrees of freedom which may have a classical part. An interaction of a quantum systems with a classical subsystem entails feedback effects which necessarily generate nonlinearities in the dynamics.

Every quantum system with charged elementary systems (like electrons) inevitably interacts with the electromagnetic radiation field. The separation of an individual object system from its electromagnetic environment leads to an individual non-autonomous subdynamics for the object subsystem with a driving force which is chaotic in the individual sense of Wiener. ${ }^{36}$ Since the electromag-

\footnotetext{
${ }^{35}$ LANDAU (1987a), LANDAU (1987b).
}

${ }^{36}$ WIENER (1930). 
netic environment acts as a K-system, ${ }^{37}$ Birkhoff's individual ergodic theorem applies, ${ }^{38}$ so that the external force acting on the object system can be regarded as an individual trajectory of a stochastic process. In this case, Birkhoff's individual ergodic theorem provides the crucial link between individual and statistical descriptions. It allows transformation of the individual nonlinear chaotic dynamics into the linear dynamics of the statistical ensemble description.

On this basis an individual description of the measuring process is feasible. In simple models the reduced dynamics is then governed by a non-autonomous nonlinear equation which transforms pure ontic states into pure ontic states. Since the reduced object system is driven by a chaotic process (in the sense of Wiener), its behavior exhibits a sensitive dependence on the initial conditions of the environment. Epistemically indistinguishable but ontically different initial states of the environment can lead to asymptotically disjoint ontic final states. In the associated statistical description, results corresponding to the mentioned model by Lockhart and Misra can be achieved.

\section{Relations between Epistemic and Ontic Descriptions}

\subsection{From first principles to observed phenomena}

No fundamental theory directly describes context-dependent phenomena of empirical science. To describe empirically accessible information in terms of a fundamental theory, the contexts introduced by measuring tools or pattern detection and recognition devices used by the experimentalist have to be taken into account explicitly. In mathematical terms, a context can be imposed on the fundamental theory by restricting its domain of validity and introducing a new, coarser topology compatible with the intrinsic topology of the underlying fundamental theory.

To get a mathematically complete and consistent theory, one has to close the derived theory in the new contextual topology. This results in a qualitatively different higher-level theory whose domain of validity may intersect nontrivially with the domain of validity of the fundamental theory. If neither one of the two domains is contained in the other, then any strong reductionist scheme fails. This situation usually leads to the emergence of qualitatively new properties.

There are many possibilities to introduce a contextual topology. A standard strategy uses singular asymptotic expansions which do not converge in the original topology of the ontic description. Examples include the emergence of phenomena of geometrical optics (such as "shadows") in the high-frequency limit, and the emergence of inductors, capacitors and resistors in the low-frequency limit of Maxwell's electrodynamics. Another instance is the emergence of the shape of molecules in the singular asymptotic Born-Oppenheimer expansion at

\footnotetext{
${ }^{37}$ Thomas (1974).

${ }^{38}$ BirkHOFF (1931).
} 
the singular point of an infinite nuclear mass. ${ }^{39}$ These and related examples show that for epistemic descriptions the specification of a context is as important as the underlying ontic description.

Another major issue in this context is the emergence of separate subsystems (parts) from an original entangled system as a whole. (Mereological emergence in this sense is different from mereological emergence in many philosophical discussions where a whole is considered to emerge from parts.) Any interaction with the system as a whole, e.g. by measurement, provides disentangled subsystems. The states of the subsystems can have properties which the entangled state before measurement did not have. If epistemic correlations between such properties violate Bell's inequality, they indicate the ontic entanglement of the original state.

A most powerful first principle in modern physics is symmetry. In the words of Weyl: "As far as I see, all a priori statements in physics have their origin in symmetry." 40 Yet, fundamental symmetries are not directly accessible by experiments. They can only be indirectly inferred by symmetry breakings.

A symmetry is spontaneously broken if the realized states show less symmetry than their associated equations of motion (or the Hamiltonian). Broken symmetries play a crucial role for the description of many physical phenomena. For example, ferromagnetism involves the spontaneous breakdown of the rotation symmetry, crystallization requires the spontaneous breakdown of the translation and rotation symmetry, superfluidity is related to the breakdown of the special Galilei symmetry, and superconductivity is connected with the spontaneously broken gauge symmetry.

At the molecular level, the parity symmetry is broken in the ground states of chiral molecules (such as L-amino acids or D-sugars). Moreover, every experiment presupposes irreversibility and requires nonanticipative measuring instruments, hence a distinction between past and future and the selection of a direction of time. Since fundamental physical laws do not distinguish between past and future, the time-inversion symmetry of the underlying fundamental ontic description has to be broken.

\subsection{Emergence in algebraic quantum theory}

In algebraic quantum theory, a particular context can be introduced by imposing a contextually selected topology upon the state space of the $\mathrm{C}^{*}$-algebra $\mathfrak{A}$ of the underlying ontic description. This new topology has to be compatible with the algebraically determined, hence context-independent norm topology of $\mathfrak{A}$. It can be implemented by a particular reference state, given by a positive linear state functional $\rho$ on the context-independent $\mathrm{C}^{*}$-algebra $\mathfrak{A}$. The so-called GNS-construction (according to Gel'fand, Naimark and Segal) then allows the construction of a context-dependent Hilbert space $\mathcal{H}_{\rho}$ and an associated faithful representation $\pi_{\rho}(\mathfrak{A})$ of the $\mathrm{C}^{*}$-algebra $\mathfrak{A}$ acting on $\mathcal{H} .{ }^{41}$ The closure of $\pi_{\rho}(\mathfrak{A})$ in

\footnotetext{
${ }^{39}$ See Primas (1998) for more details.

${ }^{40}$ WEYL (1952), p.126.

${ }^{41}$ Compare for example Takesaki (1979), or Bratteli \& Robinson (1987).
} 
the weak topology of the algebra $\mathfrak{B}\left(\mathcal{H}_{\rho}\right)$ of all bounded operators acting on $\mathcal{H}_{\rho}$ is a context-dependent $\mathrm{W}^{*}$-algebra $\mathfrak{M}_{\rho}$, called the algebra of contextual observables with respect to the contextual topology generated by the reference state functional $\rho$. The relation $\mathfrak{A} \sim \pi_{\rho}(\mathfrak{A}) \subset \mathfrak{M}_{\rho} \subset \mathfrak{B}\left(\mathcal{H}_{\rho}\right)$ implies that all intrinsic properties appear also as contextual properties, but in addition there are new contextual properties which are not intrinsic.

Observables in the $\mathrm{W}^{*}$-algebra $\mathfrak{M}_{\rho}$ of contextual observables, which are outside the representation of the $\mathrm{C}^{*}$-algebra $\pi_{\rho}(\mathfrak{A})$, are called emergent observables. They represent properties which are novel in the sense that they are absent in the more fundamental context-independent $\mathrm{C}^{*}$-algebraic description. The emergence of novelty in contextual descriptions is a compelling fact in algebraic quantum theory. Simple examples of emergent classical observables are the temperature $^{42}$ and chemical potential ${ }^{43}$ which arise in a most natural manner from a GNS-construction with respect to canonical KMS-states.

A basic common feature in all these cases of emergent behavior is the transition from an ontic to an epistemic description. If the interaction of an object system with its environment is not excluded, there are in general infinitely many physically inequivalent representations of the object system. Choosing one of these representations means to select a particular context for the epistemic description. The simplicity of natural laws manifests itself only in the ontic description, while a representation of the richness of observable phenomena requires the multitude of inequivalent representations.

\subsection{Relative onticity}

Although a picture comprising both epistemic and ontic descriptions together with their relationship is very appealing, it is still too simplistic if epistemic and ontic elements must be considered in one and the same descriptive framework. For instance, such a situation is unavoidable if, as in Bohr's perspective, an epistemic description of a system is assumed to presuppose an ontic description of measuring tools. In such situations, a combination of ontic and epistemic elements is required in the desired descriptive framework. This difficulty can be resolved if it is realized that the distinction of epistemic and ontic descriptions can be applied to the entire hierarchy of (perhaps partially overlapping) domains leading from fundamental particles in basic physics to chemistry and even to living systems in biology and psychology. Ontic and epistemic descriptions are then considered as relative to two (successive) domains in the hierarchy.

Let us briefly illustrate this by an example. From the fundamental viewpoint of quantum theory, atoms and molecules are objects with highly contextual properties, which can be described by interactions of electrons, nuclei, and their environments. However, from the viewpoint of a chemist one is usually not interested in these interactions, but in the shape, chirality, and similar features of molecules. In such a framework, it is reasonable to consider the properties

\footnotetext{
42 TAKesaki (1970).

${ }^{43}$ MülleR-Herold (1980).
} 
of an atom or a molecule as intrinsic properties in an ontic description rather than as epistemic, contextual properties derived from more basic intrinsic properties of protons, neutrons, or even "more basic" constituents. While atoms and molecules are epistemically described within the domain of basic physics, they acquire ontic significance within the domain of chemistry.

Similarly, liquid water has properties which water molecules do not have, e.g. the property of wetness. It can be derived as an emergent, contextual property from an underlying ontic description. For a chemist, however, it would be absurd to dismiss the wetness of water as something which does not refer to an "independent reality" of water. Why should water only be wet if it is observed?

The central point of the concept of relative onticity is that states and properties of a system, which belong to an epistemic description in a particular domain, can be considered as ontic from the perspective of another domain. This idea resembles the (less formal) discussion of "ontological relativity" originally introduced by Quine. ${ }^{44}$ Quine argues that if there is one ontology that fulfills a given theory, then there is more than one. He, thus, claims that it makes no sense to say what the objects of a theory are, beyond saying how to interpret or reinterpret that theory in another theory.

For Quine, any question as to the "quiddity" (the "whatness") of a thing is meaningless unless a conceptual scheme is specified relative to which it is discussed. He encourages "ontological commitment" in the sense that a most proper conceptual frame should be preferred for the interpretation of a theory. For Quine, the inscrutability of reference is the issue which causes the problems necessitating ontological relativity, not the unique assignment of referents as objects in the external world. ${ }^{45}$

Putnam has developed a related kind of ontological relativity, first called "internal realism", later sometimes modified to "pragmatic realism". 46 Ontological (sometimes conceptual) relativity in Putnam's internal realism differs from Quine's usage of the term in an important detail. While Quine's ontological relativity is due to the impossibility of a uniquely fixed relationship of our concepts to the totality of objects to which those concepts refer, Putnam's position is more radical insofar as he questions that we know what we mean when we speak of a totality of objects. ${ }^{47}$ This shift in emphasis is particularly interesting in view of the holistic features of quantum systems which challenge the notion of an object as part of a system within an ontic description.

From this rough characterization, ${ }^{48}$ a close relation between ontological relativity à la Putnam and the idea of relative onticity is apparent. Assuming that Putnam's notion of an object can be more precisely characterized by the states and properties of such an object, conceptual frames or schemes serve a purpose very similar to contextual representations in the framework of algebraic

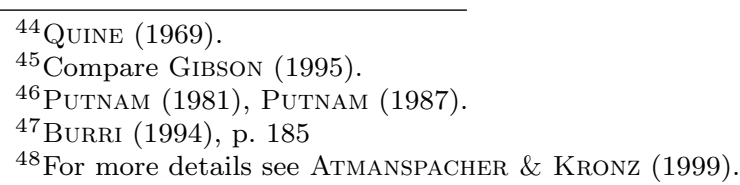


quantum theory. Both ontological relativity and relative onticity refer to a conception of realism where the states and properties of objects have to be described relative to a context, and they agree with regard to a basic assertion according to which there is a "real world as such". These basic issues can be successfully implemented in a sound formal manner, if epistemic and ontic descriptions are properly distinguished and related to each other.

\section{Conclusions}

Distinguishing epistemic and ontic descriptions of quantum systems is a key to avoid the category mistake of confounding concepts of observation-dependent and observation-independent quantum realities. Epistemic descriptions refer to a statistical description of ensembles of experimental outcomes. By contrast, ontic descriptions refer to individual systems without any respect to their observation or measurement. Epistemic and ontic descriptions require different mathematical codifications.

Although all applications of quantum theory are based on epistemic formulations, this does not imply that ontic descriptions are altogether pointless. Since they are free from particular contexts required for particular applications, ontic descriptions necessarily feature a higher degree of symmetry than epistemic descriptions. It is possible to derive epistemic descriptions from ontic descriptions, if the particular contexts can be implemented precisely enough. An ontic description of a system can give rise to many different epistemic descriptions mutually excluding each other.

An ultimately context-free ontic description or, equivalently, a universal context would be of perfect symmetry and cannot be formulated. Hence, any ontic description presupposes an "ontological commitment", an agreement about a fundamental domain of discourse based on some context which is as general as possible or reasonable. This entails a relativity of onticity which is crucial for the coherent discussion of emergent behavior and can be formalized by contextual symmetry breakings.

\section{References}

Atmanspacher, H. \& Kronz, F. (1999): Relative onticity. In: Atmanspacher, H., Amann, A. \& Müller-Herold, U. (eds.): On Quanta, Mind and Matter. Dordrecht: Kluwer. Pp.273-294.

Bell, J.S. (1975): On wave packet reduction in the Coleman-Hepp model. Helvetica Physica Acta 48, 93-98.

Berberian, S.K. (1966): Notes on Spectral Theory. Princeton: Van Nostrand.

Birkhoff, G.D. (1931): Proof of the ergodic theorem. Proceedings of the National Academy of Sciences of the United States of America 17, 656-660.

Bohr, N. (1949): Discussion with Einstein on epistemological problems in atomic physics. In: Schilpp, P. A. (ed.): Albert Einstein: Philosopher-Scientist. Evanston, Illinois: Library of Living Philosophers. Pp.199-241. 
Bratteli, O. \& Robinson, D.W (1987): Operator Algebras and Quantum Statistical Mechanics. I. $C^{*}$ - and $W^{*}$-Algebras, Symmetry Groups, Decomposition of States. Second edition. New York: Springer.

Burri, A. (1994): Hilary Putnam. Frankfurt: Campus Verlag.

Dirac, P.A.M. (1926): On the theory of quantum mechanics. Proceedings of the Royal Society (London) A112, 661-677.

Einstein, A. (1949a): Autobiographical notes. In: SchilpP, P. A. (ed.): Albert Einstein: Philosopher-Scientist. Evanston, Illinois: Library of Living Philosophers. Pp.1-95.

Einstein, A. (1949b): Reply to criticism. In: Schilpp, P. A. (ed.): Albert Einstein: Philosopher-Scientist. Evanston, Illinois: Library of Living Philosophers. Pp.665-688.

Fetzer, J.H. \& Almeder, R.F (1993): Glossary of Epistemology / Philosophy of Science. New York: Paragon House.

Gibson, R.F. (1995): Quine, Willard Van Orman. In: Kim, J. \& SosA, E. (ed.): A Companion to Metaphysics. Oxford: Blackwell. Pp.426-428.

Gleason, A.M. (1957): Measures on the closed subspaces of a Hilbert space. Journal of Mathematics and Mechanics 6, 885-893.

HePP, K. (1972): Quantum theory of measurement and macroscopic observables. Helvetica Physica Acta 45, 237-248.

Holevo, A.S. (1972): On the mathematical theory of quantum communication channels. Problems of Information Transmission 8, 47-54. Russian original: Problemy Peredači Informacii 8, (1972), pp. 63-71.

KADISON, R.V. (1965): Transformations of states in operator theory and dynamics. Topology 3, Suppl. 2, 177-198.

Kochen, S. \& Specker, E.P. (1965): Logical structures arising in quantum theory. In: Addison, J., Henkin, L. \& Tarski, A. (eds.): The Theory of Models. Amsterdam: North Holland.

Kochen, S. \& Specker, E.P. (1967): The problem of hidden variables in quantum mechanics. Journal of Mathematics and Mechanics 17, 59-88.

Koopman, B.O. (1931): Hamiltonian systems and transformations in Hilbert space. Proceedings of the National Academy of Sciences of the United States of America 17, $315-318$.

LANDAU, L.J. (1987a): On the violation of Bell's inequality in quantum theory. Physics Letters A 120, 54-56.

LANDAU, L.J. (1987b): Experimental tests of general quantum theories. Letters in Mathematical Physics 14, 33-40.

LOCKHART, C.M. \& MisRA, B (1986): Irreversibility and measurement in quantum mechanics. Physica A 136, 47-76.

Mackey, G.W. (1963): The Mathematical Foundations of Quantum Mechanics. New York: Benjamin.

Misra, B. (1967): When can hidden variables be excluded in quantum mechanics? Nuovo Cimento 47 A, 841-859.

Müller-Herold, U. (1980): Disjointness of $\beta$-KMS states with different chemical potential. Letters in Mathematical Physics 4, 45-88.

Neumann, J.von (1931): Die Eindeutigkeit der Schrödingerschen Operatoren. Mathematische Annalen 104, 570-578.

Neumann, J.von (1932): Mathematische Grundlagen der Quantenmechanik. Berlin: Springer.

Petersen, A. (1963): The philosophy of Niels Bohr. Bulletin of the Atomic Scientist 19, No. $7,8-14$. 
Primas, H. (1997): The representation of facts in physical theories. In: AtmanspaCher, H. \& Ruhnau, E. (eds.): Time, Temporality, Now. Berlin: Springer. Pp.241-263.

Primas, H. (1998): Emergence in exact natural science. Acta Polytechnica Scandinavica Ma 91, 83-98.

Primas, H. (2000): Asymptotically disjoint quantum states. In: Blanchard, P., Giulini, D., Joos, E., Kiefer, C. \& Stamatescu, I.-O. (eds.): Decoherence: Theoretical, Experimental, and Conceptual Problems. Berlin: Springer. Pp.161-178.

Putnam, H. (1981): Reason, Truth and History. Cambridge: Cambridge University Press.

Putnam, H. (1987): The Many Faces of Realism. La Salle: Open Court.

Quine, W.V. (1969): Ontological relativity. In: Quine, W. V. (ed.): Ontological Relativity and Other Essays. New York: Columbia University Press. Pp.26-68.

Roberts, J.E. \& Roepstorff, G. (1969): Some basic concepts of algebraic quantum theory. Communications in Mathematical Physics 11, 321-338.

Scheibe, E. (1964): Die kontingenten Aussagen in der Physik. Frankfurt: Athenäum Verlag. Scheibe, E. (1973): The Logical Analysis of Quantum Mechanics. Oxford: Pergamon Press.

SCHRÖDINGER, E. (1935): Die gegenwärtige Situation in der Quantenmechanik. Naturwissenschaften 23, 807-812, 823-828, 844-849.

Stone, M.H. (1930): Linear transformations in Hilbert space. III. Operational methods and group theory. Proceedings of the National Academy of Sciences of the United States of America 16, 172-175.

Stone, M.H. (1949): Postulates for the barycentric calculus. Annali di Matematica Pura ed Applicata 29, 25-30.

TAKesaki, M. (1970): Disjointness of the KMS states of different temperatures. Communications in Mathematical Physics 17, 33-41.

TAKESAKI, M. (1979): Theory of Operator Algebras I. New York: Springer.

Thomas, L.C. (1974): A note on quantising Kolmogorov systems. Annales de l'Institut Henri Poincaré, Physique théorique A 21, 77-79.

Weizsäcker, C.F. von (1985): Aufbau der Physik. München: Hanser Verlag.

Weyl, H. (1952): Symmetry. Princeton: Princeton University Press.

WiEner, N. (1930): Generalized harmonic analysis. Acta Mathematica 55, 117-258. 